\section{Does it make sense to investigate whether the offspring of people with a total knee replacement for severe primary knee osteoarthritis have a higher risk of worsening knee pain?}

We read with deep interest the article by Pan $e t a l^{1}$ related to the familial effects of total knee replacement for severe primary knee osteoarthritis (OA) on worsening knee pain over 8 years. This prospective study suggested that offspring with a family history of knee OA had an increased risk of worsening knee pain independent of some confounding factors. Including several other related researches published previously, ${ }^{2-5}$ we really appreciate the work that has been done by the authors. The genetic contributions to knee pain, radiographic knee OA, muscle strength, cartilage volume and bone size have been fully realised; however, some worthwhile issues need to be explored.

The authors compared offspring having at least one parent with a total knee replacement for severe primary knee OA with controls, selected at random from the state electoral roll (2000), without a history of knee OA in either parent. But the outcome was risk of worsening knee pain, not incidence or progression of knee OA. It is worth debating. As we all know, there is an ongoing debate on whether an association between radiographic and clinical OA exists, ${ }^{6}$ especially for early OA. There is the possibility that early knee OA patients feel no pain. More certain is the fact that a substantial part of knee pain is due to other reasons, not just knee OA. At this point, the authors admitted that knee pain might result from other musculoskeletal diseases or other sites of the body, but they had not screened for these conditions. We are not sure whether it is better to examine the genetic contribution to the incidence or progression of knee OA. The results of these outcomes are worthy of expectation. In addition, logistic regression modelling was used to assess the potential relationships between the status of participants and change in knee pain after adjustment for several confounders including injury. However, knee injury is more than just a confounding factor. It is thought to result in knee pain directly. We suggest that participants with knee injury be excluded. Furthermore, we are confused about the title with respect to 'worsening knee pain'. It seems like every participant at baseline has felt knee pain, but the fact is, not. Also, we are interested in the effects of genetic contribution to the incidence and progression of knee pain respectively. We are very much looking forward to the results of such subgroup analyses.

In addition to all the above, there are some other issues that need to be mentioned. First, the authors indicated that a higher prevalence of knee pain was found in people with at least one parent undergoing a total knee replacement for severe primary knee $\mathrm{OA}$ at baseline in their previous cross-sectional report. ${ }^{2}$ However, it was confirmed that the study design was a population-based case-control study. Second, did the authors have information on the treatment of pain or knee OA during the follow-up period? These might have affected the results, especially in regard to painkillers. Third, the sample size of this prospective study was small. How was the sample size calculated? Fourth, follow-up rate was less than $60 \%$,but the authors did not report the reasons of withdrawal. Lastly, there should be some other confounders that need to be addressed, such as exercise type and intensity, occupation, alcohol-drinking status, diabetes mellitus, hypertension, etc.

We respect the great contributions of the authors, and we would also be very interested in the authors' response on these issues.

\section{Chao Zeng, ${ }^{1}$ Jie Wei, ${ }^{2}$ Guang-hua Lei ${ }^{1}$}

${ }^{1}$ Department of Orthopaedics, Xiangya Hospital, Central South University, Changsha, Hunan Province, China

${ }^{2}$ Department of Epidemiology and Health Statistics, School of Public Health, Central South University, Changsha, Hunan Province, China

Correspondence to Professor Guang-hua Lei, Department of Orthopaedics, Xiangya Hospital, Central South University, Xiangya Road, Changsha, Hunan Province, China, 410008; Igh9640@sina.cn

Contributors CZ: concept, writing; JW: writing; G-hL: concept, revising. Competing interests None.

Provenance and peer review Commissioned; internally peer reviewed.

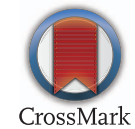

To cite Zeng C, Wei J, Lei G-hua. Ann Rheum Dis 2015;74:e44.

Received 16 March 2015

Accepted 19 March 2015

Published Online First 15 April 2015

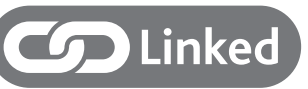

http://dx.doi.org/10.1136/annrheumdis-2015-207620

Ann Rheum Dis 2015;74:e44. doi:10.1136/annrheumdis-2015-207603

\section{REFERENCES}

1 Pan F, Ding C, Winzenberg $T$, et al. The offspring of people with a total knee replacement for severe primary knee osteoarthritis have a higher risk of worsening knee pain over 8 years. Ann Rheum Dis 2014. Published Online First: 4 Dec 2014. doi:10.1136/annrheumdis-2014-206005

2 Jones G, Ding C, Scott F, et al. Genetic mechanisms of knee osteoarthritis: a population based case-control study. Ann Rheum Dis 2004;63:1255-9.

3 Zhai G, Stankovich J, Ding C, et al. The genetic contribution to muscle strength, knee pain, cartilage volume, bone size, and radiographic osteoarthritis: a sibpair study. Arthritis Rheum 2004;60:628-9.

4 Ding C, Cicuttini F, Scott F, et al. Association between age and knee structural change: a cross sectional MRI based study. Ann Rheum Dis 2005;64:549-55.

5 Ding C, Cicuttini F, Blizzard L, et al. Genetic mechanisms of knee osteoarthritis: a population-based longitudinal study. Arthritis Res Ther 2006;8:R8.

6 Kinds MB, Welsing PM, Vignon EP, et al. A systematic review of the association between radiographic and clinical osteoarthritis of hip and knee. Osteoarthritis Cartilage 2011;19:768-78. 